\title{
Feasibility Study for Applying Spectral Imaging for Wheat Grain Authenticity Testing in Pasta
}

\author{
Timothy Wilkes ${ }^{1}$, Gavin Nixon ${ }^{1}$, Claire Bushell ${ }^{1}$, Adrian Waltho², Amer Alroichdi ${ }^{3}$, \\ Malcolm Burns ${ }^{1^{*}}$ \\ ${ }^{1}$ Science and Innovation, LGC Ltd., Teddington, UK \\ ${ }^{2}$ Analytik Ltd., Cambridge, UK \\ ${ }^{3}$ Mapping Solutions Ltd., Manchester, UK \\ Email: *malcolm.burns@lgcgroup.com
}

Received 19 February 2016; accepted 25 April 2016; published 28 April 2016

Copyright (C) 2016 by authors and Scientific Research Publishing Inc.

This work is licensed under the Creative Commons Attribution-NonCommercial International License (CC BY-NC).

http://creativecommons.org/licenses/by-nc/4.0/

(c) () (9) Open Access

\section{Abstract}

Authentication of pasta is currently determined using molecular biology-based techniques focusing on DNA as the target analyte. Whilst proven to be effective, these approaches can be criticised as being destructive, time consuming, and requiring specialist instrument training. Advances in the field of multispectral imaging (MSI) and hyperspectral imaging (HSI) have facilitated the development of compact imaging platforms with the capability to rapidly differentiate a range of materials (inclusive of grains and seeds) based on surface colour, texture and chemical composition. This preliminary investigation evaluated the applicability of spectral imaging for identification and quantitation of durum wheat grain samples in relation to pasta authenticity. MSI and HSI were capable of rapidly distinguishing between durum wheat and adulterant common wheat cultivars and assigning percentage adulteration levels characterised by low biases and good repeatability estimates. The results demonstrated the potential for spectral imaging based seed/grain adulteration testing to augment existing standard molecular approaches for food authenticity testing.

\section{Keywords}

Spectral Imaging, Multispectral Imaging, Hyperspectral Imaging, Durum Wheat Authentication, Rapid Non-Destructive Testing, Food Adulteration

\footnotetext{
${ }^{*}$ Corresponding author.
}

How to cite this paper: Wilkes, T., Nixon, G., Bushell, C., Waltho, A., Alroichdi, A. and Burns, M. (2016) Feasibility Study for Applying Spectral Imaging for Wheat Grain Authenticity Testing in Pasta. Food and Nutrition Sciences, 7, 355-361. 


\section{Introduction}

Recent advances in the field of spectral imaging have facilitated the development of a number of compact, multispectral and hyperspectral imaging platforms with the capability to differentiate between surface colour, texture and chemical composition for a range of materials [1]. The non-destructive nature of these instruments, decreased requirement for expensive consumables, and suitability for automatic image/data analyses which minimises the need for specialised training, means that these systems have the potential to be used for point of test approaches and screening applications.

Multispectral imaging (MSI) has been widely applied to the classification of seed and grains, including maize, wheat, barley, soybean and rice. Recent publications in the scientific literature have described the use of spectral imaging as a means to distinguish between hard, intermediate and soft maize kernels [2] [3], for the discrimination of grain from different maize varieties [3], and between different cultivars of rice [4].

For the manufacture of pasta, current EU law allows up to 3\% (w/w) adventitious contamination with other wheat (T. aestivum or common wheat) [5]. Authenticity of pasta samples is typically determined using molecular biology techniques which focus on DNA as the target analyte [6]. Whilst proven to be relatively accurate, current DNA approaches for testing for food adulteration often require relatively laborious upstream and complex sample preparation phases inclusive of destructive DNA extraction procedures [7] [8].

Spectral imaging represents a technology that can be used for quality and safety testing of foods [9], but also has potential application in food authenticity testing, based on utilisation of the closely related multispectral and hyperspectral imaging techniques. Multispectral imaging (MSI) and hyperspectral imaging (HSI) differ mainly on the complexity of spectral data collected: essentially hyperspectral systems measure energy in narrower and more numerous bands than multispectral systems. Spectral imaging offers potential benefits over existing DNA analytical approaches including time, processing (i.e. non-destructive), sample throughput, and no requirement for specialised training or expensive reagents/consumables [10].

This work describes a preliminary investigation into the application and evaluation of performance characteristics of spectral imaging for food authenticity testing. This feasibility study focused on investigating spectral imaging to reliably distinguish between $T$. durum and T. aestivum wheat grains (which are important in the production of pasta) based on the spectral signature of each grain species, using a panel of deliberately adulterated wheat samples designed to be at or close to the current EU legislative limit. Spectral imaging performance was evaluated on the basis of the ability to correctly assign the percentage level of adulteration of the wheat test samples based on trueness and precision estimates.

\section{Materials and Methods}

\subsection{Wheat Samples}

Authenticated wheat grain samples for both Triticum aestivum (Solstice Wheat variety) and Triticum durum (French Durum Wheat variety) wheat cultivars were provided by Frontier Agriculture Ltd. (Diss, UK).

\subsection{Evaluation Study Design}

A panel of 23 wheat test samples (2 calibration controls, 21 test mixtures) were prepared which represented levels of adulteration at and around the current European Union legislative limit for labelling as per Regulation (EC) No 1222/94 [5] for pasta production. Whilst the current EU legislation expresses adulteration levels in terms of weight for weight (w/w), permitting up to $3 \%(\mathrm{w} / \mathrm{w}) T$. aestivum adulteration of $T$. durum, a durum wheat adulteration test panel was prepared on a grain/grain basis due to the non-destructive nature of the analyses that interrogated the sample at the individual grain level.

The panel comprised seven levels of sample adulteration, which represented $0 \%, 0.5 \%, 2 \%, 3 \%, 5 \%, 10 \%$, and $100 \%$ (grain/grain) of T. aestivum in total grain (T. aestivum and T. durum). Each level was represented by triplicate samples, with each sample being comprised of 100 grains with the exception of the $0.5 \%$ (grain/grain) test sample which was comprised of 200 grains (one grain of T. aestivum in a background of 199 grains of $T$. durum). These 21 test mixtures were randomised and then analysed blindly by the spectral imaging instruments. Positive Control samples for T. aestivum and T. durum were also provided, consisting of 300 grains of each.

Samples were prepared by manually transferring appropriate numbers of individual wheat grains to a Petri dish (90 mm diameter) using tweezers and the samples then stored in re-sealable plastic bags. Sample composi- 
tion was monitored using a hand held tally counter to ensure accurate grain numbers during the preparative process and to confirm total grain numbers. The order of the samples was randomised and samples were analysed blindly to ensure further confidence in the results.

\subsection{Spectral Imaging Instruments}

Two commercial spectral imaging instruments were evaluated that comprised the VideometerLab 2 (Videometer A/S, Hørsholm, Denmark), a MSI system distributed by Analytik Ltd (Cygnus Business Park, Cambridge, UK), and the NEO HySpex VNIR-1600 (Norsk Elektro Optikk A/S, Lørenskog, Norway), a HSI system distributed by Mapping Solutions Ltd. (Long Sight Business Park, Manchester, UK).

\subsection{VideometerLab 2-Based Analyses}

Each sample of wheat grain was emptied into a standard $90 \mathrm{~mm}$ diameter Petri dish before being placed under the VideometerLab 2 (Videometer A/S, Hørsholm, Denmark) integrating sphere and image data captured using VideometerLab Software Revision: 2.13.15 (3088).

Data analysis was performed using VideometerLab Software Revision: 2.13 .89 (3475) which combined object separation and image analysis capabilities. Initially, the wheat Positive Control samples (100\% T. aestivum and $100 \%$ T. durum) were imaged and the data used to construct a normalised-Canonical Discriminant Analysis model (nCDA) [11] [12] for use in the analysis of the test samples. Individual wheat grains in the test sample mixtures were identified and separated by threshold and morphology segmentation in the software, which then applied the nCDA-based model to all of the pixels falling within the boundaries of each individual wheat grain. Each grain was represented by thousands of individual pixels, and each pixel was scored on an arbitrary scale as to whether it matched the spectral signature of T. aestivum or T. durum more closely. An algorithm was then applied to calculate the likelihood of each grain being T. aestivum or $T$. durum based on composite spectral signature of the pixels within each grain.

For an individual grain, the area fraction of its pixels that were classified as T. durum determined the score for that grain. The software then ordered the grains by increasing score to calculate the number of $T$. durum and $T$. aestivum grains present and reported the results in tabular form.

\subsection{HySpex VNIR-1600-Based Analyses}

A HySpex VNIR-1600 camera (Norsk Elektro Optikk A/S, Lørenskog, Norway), utilising the VNIR hyperspectral camera functionality $(400-1000 \mathrm{~nm})$ with $1 \mathrm{~m}$ focal length lenses, was mounted on a aluminum test rack $100 \mathrm{~cm}$ above the samples, two 500W lamps placed at the same heights and the light source focused on the centre of the sample area. The viewing angle of the imaging spectrometer was set to $17^{\circ}$, spectral sampling set at 3.7 $\mathrm{nm}$ and the number of bands set at 160 .

A panel of calibration samples was generated using the $100 \%$ T. durum and T. aestivum Positive Control materials to reflect a range of $T$. aestivum adulteration. All wheat grain samples were then placed on a black moving plate for imaging, and spectra captured using the HySpexVNIR-1600 imaging spectrometer (Norsk Elektro Optikk A/S, Lørenskog, Norway). Samples were imaged alongside grey calibrated Spectralon ${ }^{\circledR}$ Target (Labsphere, Inc., North Sutton, US) in order to allow levels of reflectance to be taken into account during subsequent stages of image processing. Data analysis was performed using ENVI Spectral image processing software (Exelis Visual Information Solutions UK) employing spectral angle mapper classification (SAM), a supervised classification method based on a spectral signature matching method developed by Boardman [13].

\section{Results}

\subsection{VideometerLab 2}

The $100 \%$ T. durum and T. aestivum Positive Control samples were used to generate a normalised-Canonical Discriminant Analysis (nCDA) transformational model based on their respective spectral signatures. The model was applied to the panel of 21 blind test samples, and results were returned based on the model's scoring of how closely the spectral signature of each grain in an image matched either the $T$. durum or T. aestivum model (Figure 1). The quantitative data (Figure 2) showed close agreement with the assigned percentage adultera- 

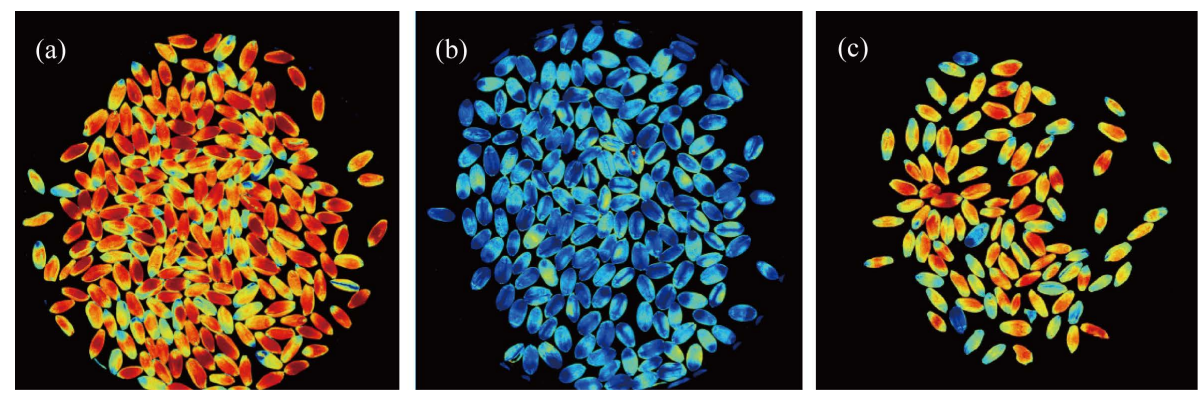

Figure 1. Example images generated using the VideometerLab 2 system for: (a) $100 \%$ T. durum wheat grains; (b) $100 \%$ T. aestivum wheat grains; (c) $10 \%$ adulteration of T. durum wheat grains with $T$. aestivum wheat grains.

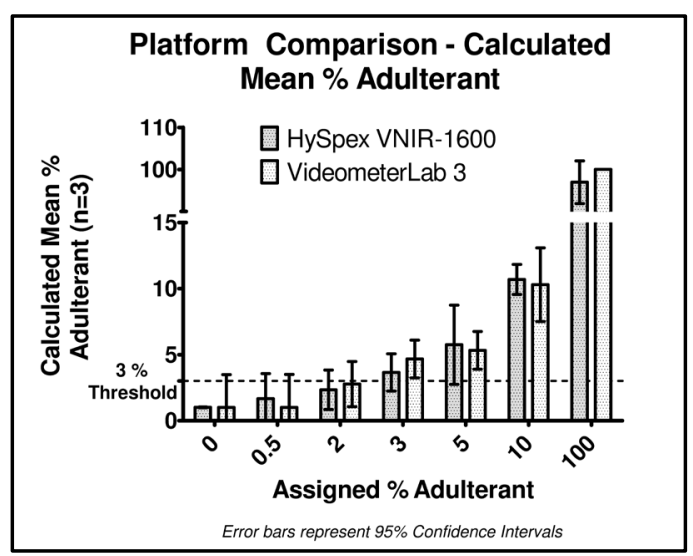

Figure 2. MSI platform comparison data displaying calculated mean percentage adulteration levels.

tion values, with lower precision at the lowest percentage adulteration samples as expected. The legislatively important 3\% adulteration sample showed a small positive bias (sample estimated as $4.67 \%$ with a ninety-five percent confidence interval of $\pm 1.43 \%$ ) with an associated Coefficient of Variation (CV) of $12.37 \%$.

\subsection{HySpexVNIR-1600}

Image processing of the 21 blind test samples was undertaken using a spectral angle mapper classification (SAM) based approach to generate the required spectral classification model as shown in Figure 3. The experimental data showed close agreement with the assigned percent adulteration values (Figure 2). Analyses of the legislatively important 3\% adulteration sample type showed a small positive bias (sample estimated as 3.65\% with a ninety-five percent confidence interval of $\pm 1.41 \%$ ), giving an associated Coefficient of Variation (CV) of $15.50 \%$.

\section{Discussion}

Based on visual acuity alone, it is very hard to reliably tell the difference between the two cultivars of $T$. aestivium and T. durum cultivars used in this study, when they are present as a mixed sample. Based on standard practice, accurate determination of the amount of adulteration in a $T$. durum sample can usually only be achieved using real-time PCR approaches, but the use of spectral imaging has provided some evidence that an alternative non-destructive analytical approach can generate reliable estimates.

The VideometerLab 2 (MSI platform) and HySpex VNIR-1600 (HSI platform) spectral imaging instruments displayed comparable quantitative performance characteristics (Figure 2) as demonstrated by similar calculated mean percentage adulterant values and associated precision data (\% CV) associated with the panel of 21 test samples. A small positive bias associated with the calculated values is observed, however the associated measurement uncertainty estimates still include the assigned values. Low-level percentage adulteration samples (e.g. 

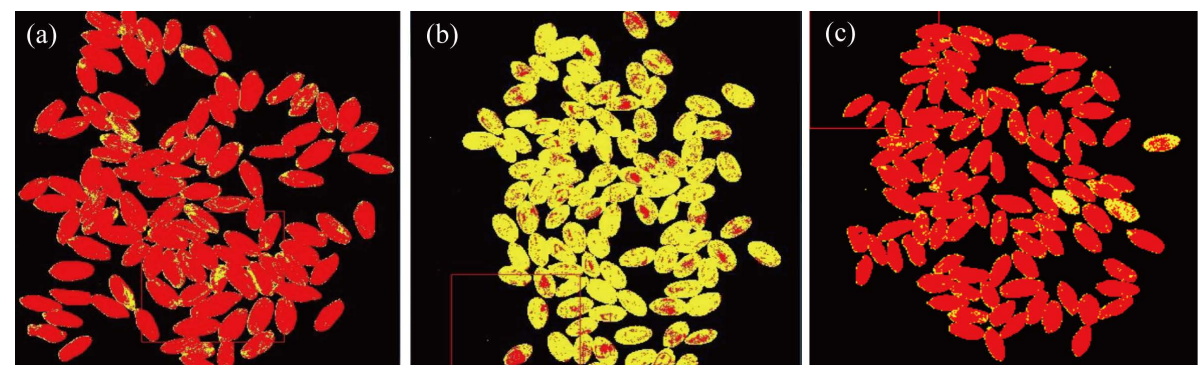

Figure 3. Example images generated using the HySpex VNIR-1600 instrument and ENVI software (SAM-based analyses). (a) 100\% T. durum sample, (b) 100\% T. aestivum sample and (c) $3 \%$ adulteration sample.

$0.5 \%$ grain/grain) appear to be more challenging for both instruments as demonstrated by the larger measurement uncertainty estimates (95\% confidence interval associated with the mean estimated value). Given the analytical uncertainty that has been reported at this level using the accepted standard of real-time PCR approaches for quantitation of durum wheat adulteration [14] (values of $5.89 \pm 3.86 \%$ and $3.0 \pm 1.6 \%$ based on experimental and extrapolated data respectively) the larger uncertainty estimate was not un-expected and is comparable to that of real-time PCR.

Quantitative performance at the legislatively important 3\% adulteration range was found to be good as characterised by acceptable bias estimates and repeatability levels ( $<16 \%$ Coefficient of Variation). These performance characteristics compare well with those reported in the published literature using real-time PCR evaluation of durum wheat samples [14]. The study findings support the conclusion that spectral imaging shows good potential for reliably distinguishing between $T$. durum and T. aestivum grain types and for quantifying potential adulteration levels. Although the selected instruments utilise alternative technical approaches, comparable results were generated which supports the earlier findings of Cocchi et al. [10] who successfully demonstrated the application of NIR spectroscopy to wheat adulteration testing based on ground flour samples. This highlights the diagnostic potential of spectroscopic techniques for seed/grain-based analyses.

The experimental model presented within this study is based on a grain for grain basis, rather than a weight for weight format referred to in the legislation, and small differences may arise between these two approaches due to natural variations in grain size and the associated impact on total grain material weight. However, whilst this approach may impact upon measurement uncertainty levels slightly, the grain per grain measurement unit helps control for heterogeneity and Poisson effects potentially associated with trace adulteration sample types, and allowed for accurate preparation of the blinded test samples. The closeness in agreement between the results generated from spectral imaging and the standard real-time PCR analysis of durum wheat samples further supported the applicability of spectral imaging as a non-destructive analytical approach. Further work is required to establish the equivalency of grain/grain-based approaches and their place within the EU legislative system for non-destructive analytical systems. However, the work presented here provides some evidence of the applicability of spectral imaging to be used as a rapid and cost effective screening approach for testing grain shipments for potential adulteration, whereby any suspect samples could then be further analysed using real-time PCR for confirmation of any such adulteration.

This feasibility study was conducted under prescriptive and controlled conditions using just two cultivars to represent the $T$. aestivum and $T$. durum wheat varieties. It is recommended that further work be conducted in order to evaluate the applicability of spectral imaging for determining differences based on more genotypes, cultivation practices, weather conditions, post-harvest storage conditions, etc. Such further work, inclusive of additional replicates, samples and grain types, could be used to extend and challenge the findings described in this preliminary investigation.

Unlike the pure grain samples employed in this study, real samples are likely to contain a range of impurities such as chaff and broken grain fragments. In these instances, analytical performance could likely be improved by including distinguishing physical characteristics into the models employed for discrimination. Further research is required to improve the analytical model which would benefit from including both spectral and spatial data for a more accurate, robust result.

The two spectral imaging-based instruments evaluated in the current study represent a new area of technolo- 
gical development with demonstrated applicability within the food testing sector. Unlike officially recognised protein [15] and DNA-based testing methods [6] [16] [17], a spectral imaging-based approach does not require complex sample processing (as it is non-destructive) or detection assays (with associated equipment) due to the use of spectral data derived from the sample to be interrogated. Other potential benefits associated with this approach for food authenticity testing include short testing times and high potential sample throughput, making the approach suited for in-line monitoring found within today's food production industry. The small foot print and lack of requirement for specialist consumables/reagents associated with some spectral imaging platforms also means they may be amenable for use as point of test approaches.

\section{Conclusions}

This initial feasibility study provides some evidence of the potential of spectral imaging techniques to be used for the authentication of durum wheat grains, through comparison to standard PCR-based approaches. Both the MSI and HSI approaches were demonstrated to be capable of rapidly distinguishing between durum wheat and adulterant common wheat. The results obtained were characterised by low biases and good repeatability estimates which compare well with the data published using real-time PCR [14].

Whilst spectral imaging offers key advantages over alternative methodologies such as its non-destructive nature and suitability for high throughput analyses, further work should be conducted to evaluate additional performance characteristics (e.g. the Limit of Detection) and applicability to other food matrices. This will provide additional evidence of the applicability of spectral imaging to be used as a rapid screening approach for food authenticity testing.

\section{Acknowledgements}

The authors gratefully acknowledge funding provided through the UK Department for Environment, Food \& Rural affairs as part of the Defra project FA0136 "Feasibility study for using rapid and automated spectral imaging for food authenticity testing" and for funding through the UK Department for Business, Innovation \& Skills as part of the Government Chemist Programme 2014-2017. The authors also thank Frontier Agriculture Ltd. (Diss, Norfolk, UK) for their kind provision of authenticated wheat samples for use in the current study, and Videometer A/S (Hørsholm, Denmark) for providing access to a VideometerLab 2 instrument.

\section{References}

[1] Dai, Q., et al. (2014) Advances in Feature Selection Methods for Hyperspectral Image Processing in Food Industry Applications: A Review. Critical Reviews in Food Science and Nutrition, 55, 1368-1382.

[2] Williams, P. and Sobering, D. (1993) Comparison of Commercial Near Infrared Transmittance and Reflectance Instruments for Analysis of Whole Grains and Seeds. Journal of Near Infrared Spectroscopy, 1, 25-32. http://dx.doi.org/10.1255/jnirs.3

[3] Zhang, X.L., Liu, F., He, Y. and Li, X.L. (2012) Application of Hyperspectral Imaging and Chemometric Calibrations for Variety Discrimination of Maize Seeds. Sensors (Basel), 12, 17234-17246. http://dx.doi.org/10.3390/s121217234

[4] Kong, W., et al. (2013) Rice Seed Cultivar Identification Using Near-Infrared Hyperspectral Imaging and Multivariate Data Analysis. Sensors (Basel), 13, 8916-8927. http://dx.doi.org/10.3390/s130708916

[5] The Commission of the European Communities, (1994) COMMISSION REGULATION (EC) No 1223/94. Official Journal of the European Communities, L136, 4.

[6] Terzi, V., et al. (2003) Development of Analytical Systems Based on Real-Time PCR for Triticum Species-Specific Detection and Quantitation of Bread Wheat Contamination in Semolina and Pasta. Journal of Cereal Science, 38, 8794. http://dx.doi.org/10.1016/S0733-5210(02)00138-8

[7] Torp, A.M., et al. (2006) Specific, Semi-Quantitative Detection of the Soybean Allergen Gly m Bd 30K DNA by PCR. Food Control, 17, 30-36. http://dx.doi.org/10.1016/j.foodcont.2004.08.005

[8] Gryson, N. (2010) Effect of Food Processing on Plant DNA Degradation and PCR-Based GMO Analysis: A Review. Analytical and Bioanalytical Chemistry, 396, 2003-2022. http://dx.doi.org/10.1007/s00216-009-3343-2

[9] Huang, H., Liu, L. and Ngadi, M.O. (2014) Recent Developments in Hyperspectral Imaging for Assessment of Food Quality and Safety. Sensors (Basel), 14, 7248-7276. http://dx.doi.org/10.3390/s140407248

[10] Cocchi, M., et al. (2006) Durum Wheat Adulteration Detection by NIR Spectroscopy Multivariate Calibration. Talanta, 
68, 1505-1511. http://dx.doi.org/10.1016/j.talanta.2005.08.005

[11] Cooley, V.W. and Lohnes, P.R. (1971) Multivariate Data Analysis. John Wiley \& Sons, N.Y.

[12] Bartlett, M.S. (1938) Further Aspects of the Theory of Multiple Regression. Mathematical Proceedings of the Cambridge Philosophical Society, 34, 33-40. http://dx.doi.org/10.1017/S0305004100019897

[13] Boardman, J.W. (1998) Leveraging the High Dimensionality of AVRIS Data for Improved Sub-Pixel Target Un-Mixing and Rejection of False Positives: Mixture Tuned Matched Filtering. 7th JPL Airborne Geoscience Workshop, Pasadena.

[14] Wiseman, G., Bowler, P. and Ohara, K. (2004) Q01085: Validation of a Protocol to Determine the Amount of NonDurum Wheat in Past. Food Standards Agency.

[15] Resmini, P. and De Bernardi, G. (1976) A Rapid Electrophoretic Method for Detecting soft Wheat in Durum Wheat, Semolina and Pasta Products. Tecnica Molitoria International, 27, 97-109.

[16] Ibrahim, M.A., et al. (2011) Detection of Durum Wheat Pasta Adulteration in the Jordanian Market by Polymerase Chain Reaction Technology. American Journal of Food Technology, 6, 492-499. http://dx.doi.org/10.3923/ajft.2011.492.499

[17] Terzi, V., et al. (2005) DNA-Based Methods for Identification and Quantification of Small Grain Cereal Mixtures and Fingerprinting of Varieties. Journal of Cereal Science, 41, 213-220. http://dx.doi.org/10.1016/j.jcs.2004.08.003 